\title{
In-Frame Deletion and Missense Mutations of the C-Terminal Helicase Domain of SMARCA2 in Three Patients with Nicolaides-Baraitser Syndrome
}

\author{
D. Wolffa $\quad$ S. Endele ${ }^{a} \quad$ S. Azzarello-Burrib $\quad$ J. Hoyer ${ }^{a} \quad$ M. Zweier ${ }^{a} \quad$ I. Schanze ${ }^{a}$ \\ B. Schmitt ${ }^{c} \quad$ A. Rauch ${ }^{b} \quad$ A. Reis ${ }^{a} \quad$ C. Zweier ${ }^{a}$ \\ ${ }^{a}$ Institute of Human Genetics, Friedrich-Alexander-University Erlangen-Nuremberg, Erlangen, Germany; \\ ${ }^{b}$ Institute of Medical Genetics, University of Zurich, Schwerzenbach, and 'Department of Pediatric Neurology, \\ University Children's Hospital Zurich, Zurich, Switzerland
}

\section{Key Words}

Intellectual disability $\cdot$ Nicolaides-Baraitser syndrome $\cdot$

Short stature $\cdot$ SMARCA2 $\cdot$ Sparse hair

\begin{abstract}
Using high-resolution molecular karyotyping with SNP arrays to identify candidate genes for etiologically unexplained intellectual disability, we identified a 32-kb de novo in-frame deletion of the C-terminal helicase domain of the SMARCA2 gene in a patient with severe intellectual disability, epilepsy, sparse hair, prominent joints, and distinct facial anomalies. Sequencing of the gene in patients with a similar phenotype revealed de novo missense mutations in this domain in 2 further patients, pointing to a crucial role of the SMARCA2 C-terminal helicase domain. The clinical features observed in all 3 patients are typical of Nicolaides-Baraitser syndrome, an only rarely reported syndrome with mainly moderate to severe intellectual disability. Notably, one of our patients with a p.Gly1132Asp mutation showed typical morphological features but an exceptional good development with borderline overall IQ and learning difficulties, thus expanding the phenotypic spectrum of NicolaidesBaraitser syndrome.

Copyright $\odot 2012$ S. Karger AG, Basel
\end{abstract}

During the last years, molecular karyotyping by either array-CGH or genomic profiling using SNP arrays has not only increased the diagnostic outcome in patients with intellectual disability harboring individual small chromosomal aberrations [de Vries et al., 2005; Hoyer et al., 2007] but also enabled the identification of several novel common microdeletion syndromes as reviewed by Slavotinek [2008]. Furthermore, it has also been proven to be a suitable method to identify novel disease-causing genes by initially identifying small aberrations affecting only one or few genes thus pointing to candidate genes in which subsequently mutations are identified in patients with similar phenotypes but without copy number variants. This strategy has been successful for well-known syndromic disorders such as autosomal-dominant CHARGE syndrome (MIM\#214800) [Vissers et al., 2004] or autosomal recessive Peters Plus syndrome (MIM\#261540) [Lesnik Oberstein et al., 2006] but also for relatively unknown entities such as Pitt-Hopkins syndrome (MIM\#610954) [Amiel et al., 2007; Zweier et al., 2007], a recognizable severe intellectual disability syndrome that only came to a broad clinical awareness after identification of the underlying gene defect. Further examples are the identification of causative

\section{KARGER}

Fax +4161306 1234

E-Mail karger@karger.ch

www.karger.com
(C) 2012 S. Karger AG, Basel

$1661-8769 / 11 / 0026-0237 \$ 38.00 / 0$

Accessible online at:

www.karger.com/msy
Christiane Zweier

Institute of Human Genetics

University Erlangen-Nuremberg, Schwabachanlage 10

DE-91054 Erlangen (Germany)

Tel.+499131852 9113, E-Mail christiane.zweier@uk-erlangen.de 


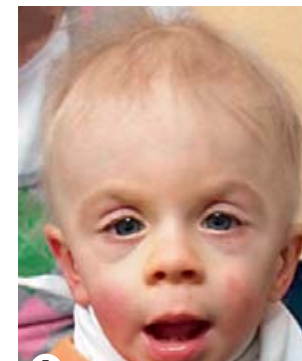

A
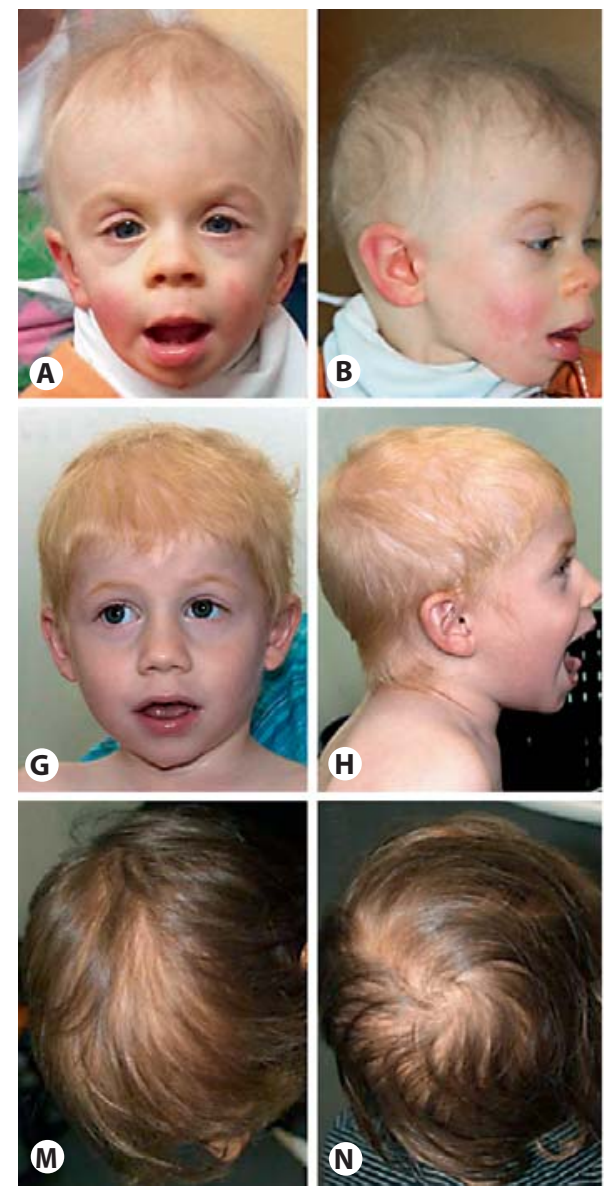
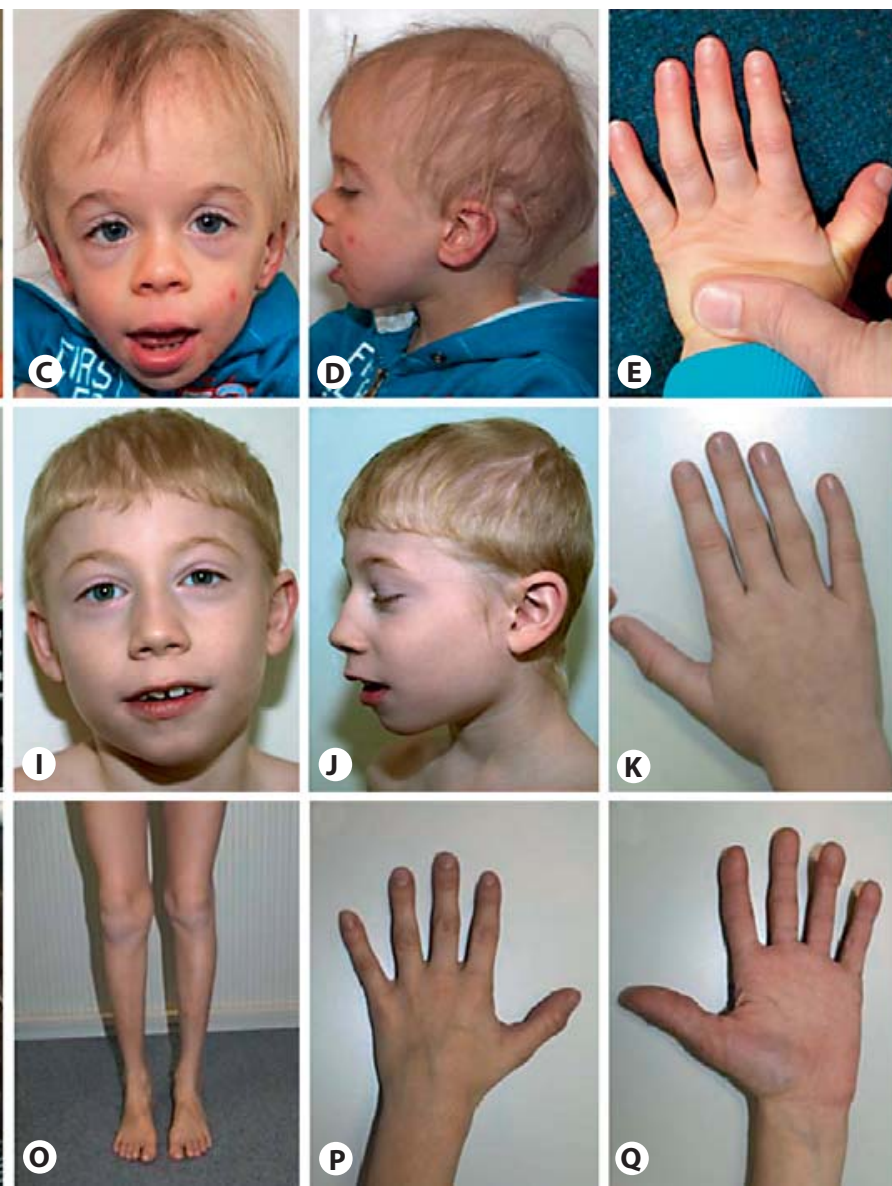

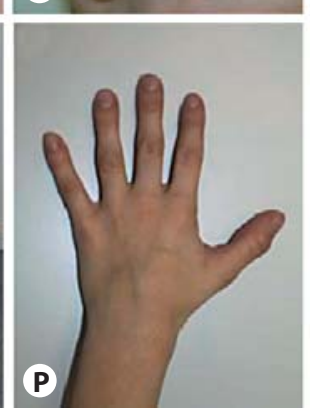

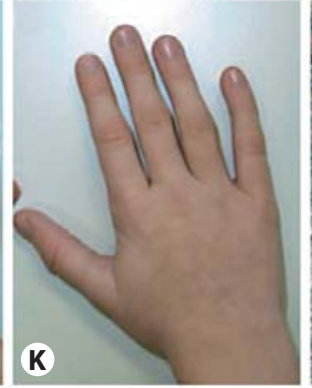

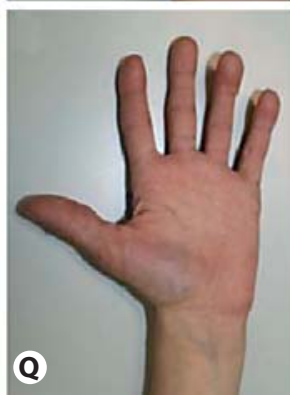

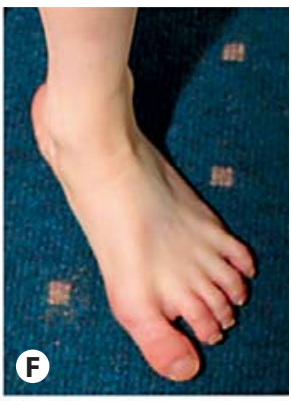
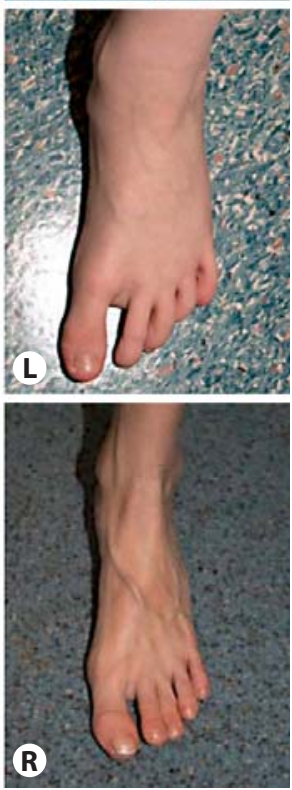

Fig. 1. Phenotypes of 3 patients with defects in SMARCA2. A, B Patient 1 at the age of 21 months. C-F Facial gestalt, hand and foot of patient 1 at the age of 3 years and 7 months. G, $\mathbf{H}$ Patient 2 at the age of 3 years and 6 months. I-L Facial gestalt, hand and foot of patient 2 at the age of 8 years. M-R Scalp hair, knees, hand from in- and outside, and foot of patient 3 at the age of 9 years.

genes for an epileptic encephalopathy [Saitsu et al., 2008] or non-specific severe intellectual disability [Zweier et al., 2010].

In our systematic endeavor to identify new disease genes in patients with intellectual disability by molecular karyotyping, we identified a small de novo deletion within the SMARCA2 gene in a patient with short stature, intellectual disability, and distinct anomalies resembling Nicolaides-Baraitser syndrome (MIM ID \%601358). By sequencing of patients with a similar phenotype, we additionally revealed missense mutations in the $\mathrm{C}$-terminal helicase domain in this gene in 2 further patients. Our findings concur with another recent report identifying SMARCA2 as the gene underlying Nicolaides-Baraitser syndrome [Van Houdt et al., 2011].

\section{Case Reports}

\section{Patient 1}

Patient 1 (fig. 1A-F), the third child of healthy, non-consanguineous parents, was born after an uneventful pregnancy at week 38 by cesarean section. His birth weight was $3,145 \mathrm{~g}$ (25th centile), his length was $52 \mathrm{~cm}$ (50th centile), and his head circumference was $35 \mathrm{~cm}$ (25th centile). The neonatal period was complicated by muscular hypotonia, feeding difficulties, and failure to thrive. Bilateral inguinal hernias were operated. Furthermore, mild aortic coarctation and cryptorchism were diagnosed. Developmental motor milestones were delayed with a sitting age of 13 months and a walking age of 30 months. At the age of 3 years and 4 months seizures occurred and were treated with valproate.

The patient was last seen at the age of 3 years and 7 months. He had short stature and microcephaly with a body height of 85 $\mathrm{cm}(<3$ rd centile), weight of $11 \mathrm{~kg}(<3$ rd centile), and head circumference of $47.5 \mathrm{~cm}$ ( $<3 \mathrm{rd}$ centile). At this time he presented with severe intellectual disability and could not speak any word. Be- 
havioral anomalies were short attention span and little social interaction with reduced eye contact. The boy had sparse hair, eczema of the skin, and facial dysmorphism with a high forehead, arched and medially sparse eyebrows, long eyelashes, wide and downslanting palpebral fissures, wrinkled skin below the eyes, flat and sagging malar regions, a short nose with upturned tip, a long philtrum, a thin upper vermillion, a full everted lower vermillion, and mildly posteriorly rotated ears. Fingers and toes were long and slender with prominent interphalangeal joints. Knees appeared large and prominent, too. Minor other anomalies were single palmar creases, one café-au-lait spot, and sandal gaps.

Metabolic screening as well as conventional karyotyping, IFISH testing for trisomy 8 mosaicism, and molecular karyotyping using an Affymetrix 6.0 Array on a $100-\mathrm{kb}$ resolution revealed normal results.

\section{Patient 2}

Patient 2 (fig. 1G-L) was the second child of healthy, non-consanguineous parents and was born after an uneventful pregnancy at week 41 . His weight was $2,890 \mathrm{~g}$ (10th centile), length was $49 \mathrm{~cm}$ (10th centile), and his head circumference was $32.5 \mathrm{~cm}(<3 \mathrm{rd}$ centile). During the neonatal period, mild feeding difficulties occurred, and a small preauricular tag was removed. Motor development was normal with a sitting age of 6 months and a walking age of 13 months. The boy started to speak single words at the age of 18 months, but further speech development ceased with the occurrence of seizures. EEG examination showed generalized irregular spike-wave complexes. The epilepsy was successfully treated with valproate and ethosuximide. Brain MRI at the age of 2 years was normal. At the age of 3 years, the boy was little interested in other children and showed auto-aggressive tendencies. Constipation was observed for a certain period of time.

The patient was last investigated at 8 years and 8 months and had mild short stature with a body height of $124.5 \mathrm{~cm}$ ( $3 \mathrm{rd}$ centile). His weight was $23.8 \mathrm{~kg}$ ( $3 \mathrm{rd}-10$ th centile), and he was microcephalic with a head circumference of $50.5 \mathrm{~cm}(<3 \mathrm{rd}$ centile). At that time he was reported to have a vocabulary of more than 100 single words and the ability to rarely speak 2 word sentences. His speech appeared monosyllabic and slurred. In general, he had a happy and sociable personality. However, attention span was short, and he was very focused on routines. Formal diagnosis of autism was made, and standardized IQ testing was done but revealed no valid results. Facial dysmorphism included mildly downslanting palpebral fissures, a thin upper lip, prominent incisors, mildly posteriorly rotated ears, and a high and narrow palate. He had sparse and dry scalp hair with low-set posterior and anterior hairlines and hypertrichosis at his neck and upper back. Further anomalies were long fingers, long toes with prominent joints, and sandal gaps.

Conventional karyotyping, testing for Fragile-X- and Angelman syndromes (incl. UBE3A sequencing), sequencing of the $A R X$ gene, and molecular karyotyping with an Affymetrix Genechip 6.0 Mapping SNP Array revealed normal results.

\section{Patient 3}

Patient 3 was the only child of healthy, non-consanguineous Caucasian parents aged 35 (mother) and 36 years (father) at birth. The boy was born at gestational week 37 after an uneventful pregnancy with mild maternal gestosis. Birth weight was $2,370 \mathrm{~g}$ (10th centile), birth length was $45 \mathrm{~cm}$ (10th centile), head circumference was $32 \mathrm{~cm}$ (10-25th centile), and Apgar-scores were 8-9-9. Following an uneventful neonatal period, developmental milestones were mildly delayed. He was able to turn around from the back at the age of 7.5 months, to sit at the age of 10 months, and to walk independently at the age of 19 months. The mother reported that he suffered from instable feet joints and drooling in early infancy and that he could hardly speak until a very short lingual frenum was cut. At the age of about 18 months he had surgery for an inguinal hernia and cryptorchism. He also had an umbilical hernia and a severe hallux valgus on the right side which was treated surgically. Formal IQ testing using the HAWIVA test at the age of 6 years and 3 months revealed a verbal comprehension IQ of 82, a perceptual reasoning IQ of 73, a processing speed IQ of 54, and a total IQ of 74. Notably, he had profound problems with auditory memory and attention deficit. He also had significant gross and fine motor delay with ataxic gait. His personality was very communicative and sociable, but he had problems with following demands and he had a short attention span. At the age of 7 years the boy developed seizures predominantly after falling asleep. Seizures usually started with weeping followed by head and eye deviation to the left or the right, and postictally by alternating ipsilateral hemiparesis. Other seizures were focal clonic or focal tonic, sometimes with secondary generalization. EEG showed sharp waves with variable manifestation from the left or right central or centro-median regions. Seizures were successfully treated with Sultiam (sulfonamide). MRI imaging of the brain at the age of 8 years was normal. An X-ray of the hand revealed a bone age retardation of 2 years. Due to his social behavior and dissociated developmental profile with strong verbal abilities, Williams-Beuren syndrome was considered as a differential diagnosis.

At the age of 9 years his weight was $20.6 \mathrm{~kg}$ ( $2 \mathrm{nd}$ centile), height was $124.5 \mathrm{~cm}$ (5th centile), and head circumference was $52 \mathrm{~cm}$ (23rd centile). Minor anomalies were a triangular face, thin, sparse and soft hair, dry and grayish skin with periorbital wrinkling and flat and sagging malar regions, long eyelashes, a narrow nasal bridge with a relatively bulbous nasal tip and a prominent columella, a relatively long and flat philtrum, a thin upper lip, mildly low-set, backwards rotated ears, a large but mildly retracted chin, sandal gaps, and a relatively short, broad second toe. The proximal interphalangeal joints and knees were thick with wrinkling skin. Distal phalanges of fingers and toes were broad and some had fetal pads. Fingers showed multiple supernumerary interphalangeal creases. The nails were reported to grow very slowly and had to be cut only rarely. Apart from one tooth, which was lost recently, primary dentition was still present and the voice was high. The boy suffered from frequent respiratory infections and multiple warts. During consultation, the boy was very friendly, communicative and active, and the mother reported that he was able to read, but attention span was still low. He attended a school for children with special needs. The boy also suffered from ichthyosis which was, however, also present in his otherwise healthy father.

Genetic testing included karyotyping, MLPA screening for 21 microdeletions (P245 probemix, MRC-Holland), sister-chromatid exchange testing, fragile $\mathrm{X}$ testing, and molecular karyotyping using an Affymetrix Cytogenetics 2.7M Array and revealed normal results. Maternal X-inactivation studies using the CAG repeat within exon 1 of the $A R$ gene revealed a skewing of 91\% in peripheral blood. 


\section{Methods}

Molecular Karyotyping

Molecular karyotyping in patient 1 and his parents was performed with an Affymetrix 6.0 SNP Array (Affymetrix, Santa Clara, Calif., USA) in accordance with the supplier's instructions. Copy number data were analysed with the Affymetrix Genotyping Console 3.0.2 software.

\section{$M L P A$}

MLPA probes for exons 17-20, 26, and 27 of the SMARCA2 gene (covering both isoforms NM_003070.3 and NM_139045.2) were designed according to the instructions of MRC-Holland, and the reaction was performed using the SALSA MLPA reagents EK5 (MRC-Holland, Amsterdam, The Netherlands) in patient 1 and his parents. The probemix contained additionally 6 internal control probes. Copy number values were calculated using values from 8 healthy control individuals and the Seqpilot software (JSI Medical Systems, Kippenheim, Germany). Probe sequences and conditions are available on request.

\section{Sequencing}

DNA samples from peripheral blood were tested for SMAR$C A 2$ mutations by bidirectional direct sequencing of all coding exons 2-34 including intronic flanking regions (ABI BigDye Terminator Sequencing Kit v.3; Applied Biosystems, Carlsbad, Calif., USA) using an automated capillary sequencer (ABI 3730; Applied Biosystems). Mutations were confirmed from an independent PCR and tested in the parents. Primer sequences and conditions are available on request. Sample mix-up in the patients with de novo mutations and their parents was excluded by using the Powerplex 16 kit according to the supplier's instructions (Promega, Madison, Wisc., USA).

This study was approved by the ethics committees of the Medical Faculty, University of Erlangen-Nuremberg and by the Kantonale Ethikkommission of Zurich, and written consent was obtained from parents or guardians of the patients.

\section{Results and Discussion}

Initial diagnostic evaluation of the Affymetrix 6.0 Array data in patient 1 with consideration of aberrations $>100 \mathrm{~kb}$ showed normal results. Prompted by our finding of mutations in ARID1B as a frequent cause of severe intellectual disability [Hoyer et al., in press], we searched for smaller aberrations in related genes in our study group of 345 individuals. This approach indeed revealed in patient 1 a heterozygous $32-\mathrm{kb}$ in-frame deletion within the SMARCA2 gene affecting exons 20 to 26 coding for the C-terminal helicase domain (fig. 2A). These results were confirmed using MLPA probes located in 2 of the deleted and the flanking non-deleted exons each (data not shown). The deletion was excluded in both parents by array analysis (fig. 2A) and by MLPA (data not shown). In addition, a copy number variant of SMARCA2 was nei- ther observed in the database of genomic variants nor in our 820 in-house control samples.

SMARCA2 encodes Brahma (BRM), 1 of 2 mutually exclusive and specific ATPase subunits [Flowers et al., 2009] of the SWI/SNF complex that was first identified in yeast and is highly conserved among eukaryotes [Kingston and Narlikar, 1999]. ARID1B, haploinsufficiency of which was recently found to cause intellectual disability, is also a subunit of this complex [Halgren et al., 2011; Hoyer et al., in press]. The SWI/SNF ATP-dependent chromatin remodeling complex regulates gene expression by using energy of ATP hydrolysis to alter chromatin structure around the regulated genes in order to facilitate access of other transcription factors [Peterson and Workman, 2000]. SMARCA2 is highly expressed in differentiating cells [Kadam and Emerson, 2003] and plays an important role in neuronal development [Yoo and Crabtree, 2009]. Loss of BRM expression in different tumor cell lines might indicate that silencing of the SWI/SNF ATPases is involved in the etiology of various tumors [Reisman et al., 2009]. A role of SNPs in SMARCA2 as susceptibility factors for schizophrenia is discussed due to respective association studies [Koga et al., 2009]. Furthermore, Smarca2 knockout mice showed impaired social interaction and prepulse inhibition [Koga et al., 2009].

Therefore, we considered the intragenic SMARCA2 deletion in patient 1 to be causative for his disorder. Given the apparently distinct phenotype of the patient, we specifically searched for similar patients seen in the genetic outpatient clinics in Erlangen and Zurich, respectively. Two patients with strong morphological resemblance but milder intellectual deficit were identified. Sequencing of SMARCA2 in these patients revealed the heterozygous missense mutation p.Ala1188Glu in exon 25 in patient 2 , and the heterozygous missense mutation p.Gly1132Asp in exon 24 in patient 3 (fig. 2B). In both patients, de novo occurrence was confirmed by excluding the respective mutation in the parents, and sampling errors were excluded by confirming probe identities within the families. Interestingly, both missense mutations were located at highly conserved amino acid positions in the C-terminal helicase domain of the SMARCA2 protein. This domain was also affected by the predicted in-frame deletion in patient 1 (fig. 2C). Therefore, our findings indicate that loss of, impaired, or altered function of the Cterminal helicase domain might underlie the common phenotypic consequences in these patients.

The striking clinical resemblance of the 3 patients includes sparse hair, borderline or mild short stature, epilepsy, and behavioral anomalies like autistic traits and 


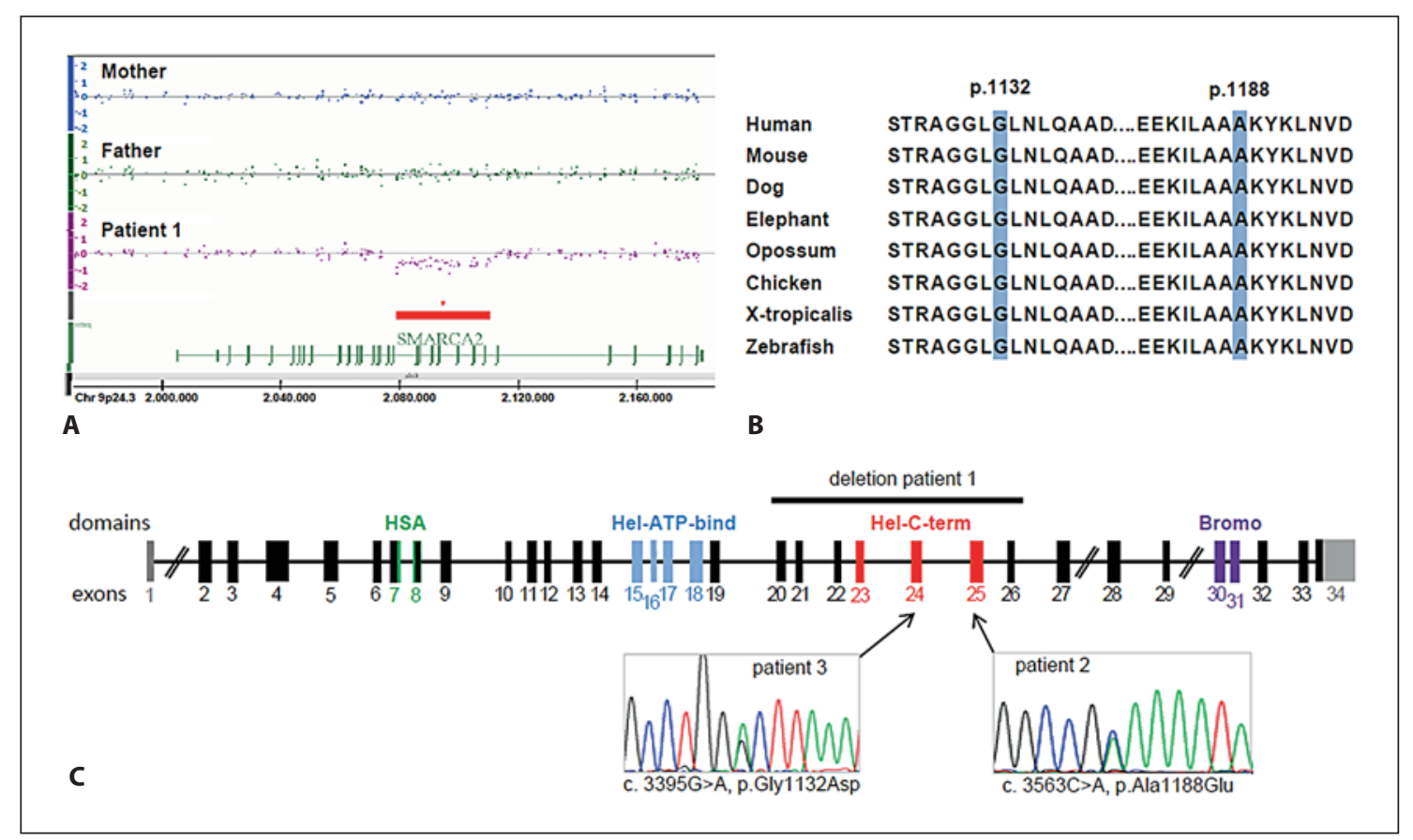

Fig. 2. Identified defects in SMARCA2. A Affymetrix 6.0 Array data of patient 1 and his parents showing an intragenic de novo deletion of $32 \mathrm{~kb}$ within the SMARCA2 gene at 9p24.3. The first and last deleted probes as indicated by the array were SNP_A-8705668 (hg18: chr9: 2,079,813) and SNP_A-8601038 (hg18: chr9: 2,111,371). B Conservation of the amino acid positions 1132 and 1188 affected by the missense mutations found in patients 2 and 3. C Schematic drawing of the SMARCA2 gene with localization of the deletion and the 2 missense mutations. Exons are color-coded for encoded domains (according to PROSITE, http://prosite.expasy.org/). HSA: helicase-SANT-associated domain; HEL-ATP-bind: helicase domain with ATP binding function; Hel-C-term: C-terminal helicase domain; Bromo: bromodomain; exon 1 and parts of exon 34 are non-coding (gray). short attention span. All 3 patients showed a distinct facial gestalt with a triangular shaped face, wide, downslanting palpebral fissures, a long philtrum, a thin upper lip, and a full or everted lower lip. Various other anomalies were microcephaly, skin anomalies such as eczema or wrinkling, hernias, long fingers and toes with prominent interphalangeal joints and broad distal phalanges, and minor congenital heart defects.

Apart from the borderline intelligence in patient 3 , all clinical features and the facial dysmorphism of our patients with SMARCA2 defects are characteristic for Nicolaides-Baraitser syndrome (table 1). After the initial description in 1993 [Nicolaides and Baraitser, 1993], this disorder was only very rarely reported until Sousa et al. [2009] published 18 new cases and delineated the clinical features of this syndrome in great detail. NicolaidesBaraitser syndrome is characterized by severe intellectual disability with absent or limited speech, short attention span, autism spectrum disorder, variable seizures, short stature, sparse hair, prominent finger joints, and distinct facial features, but it is very likely under-diagnosed since so far only 26 patients have been reported [Nicolaides and Baraitser, 1993; Krajewska-Walasek et al., 1996; Morin et al., 2003; Witters and Fryns, 2003; Castori et al., 2008; Sousa et al., 2009; Gana et al., 2011]. Until now, inheritance and underlying genetic cause were unknown, but because most patients were sporadic, autosomal dominant new mutations were suspected. Indeed, mutations in SMARCA2 were identified as the underlying cause of Nicolaides-Baraitser syndrome by whole exome sequencing and presented at the 12th International Congress of Human Genetics [Van Houdt et al., 2011].

Of note, our patient with the intragenic deletion appears to be more severely intellectually disabled than the 2 patients with missense mutations in the helicase domain. Whether this might represent a possible genotypephenotype correlation is currently unclear due to our limited number of molecularly confirmed patients and as all of our 3 patients fit into the described clinical spectrum of Nicolaides-Baraitser syndrome. In this context, 
Table 1. Clinical details of patients with Nicolaides-Baraitser syndrome

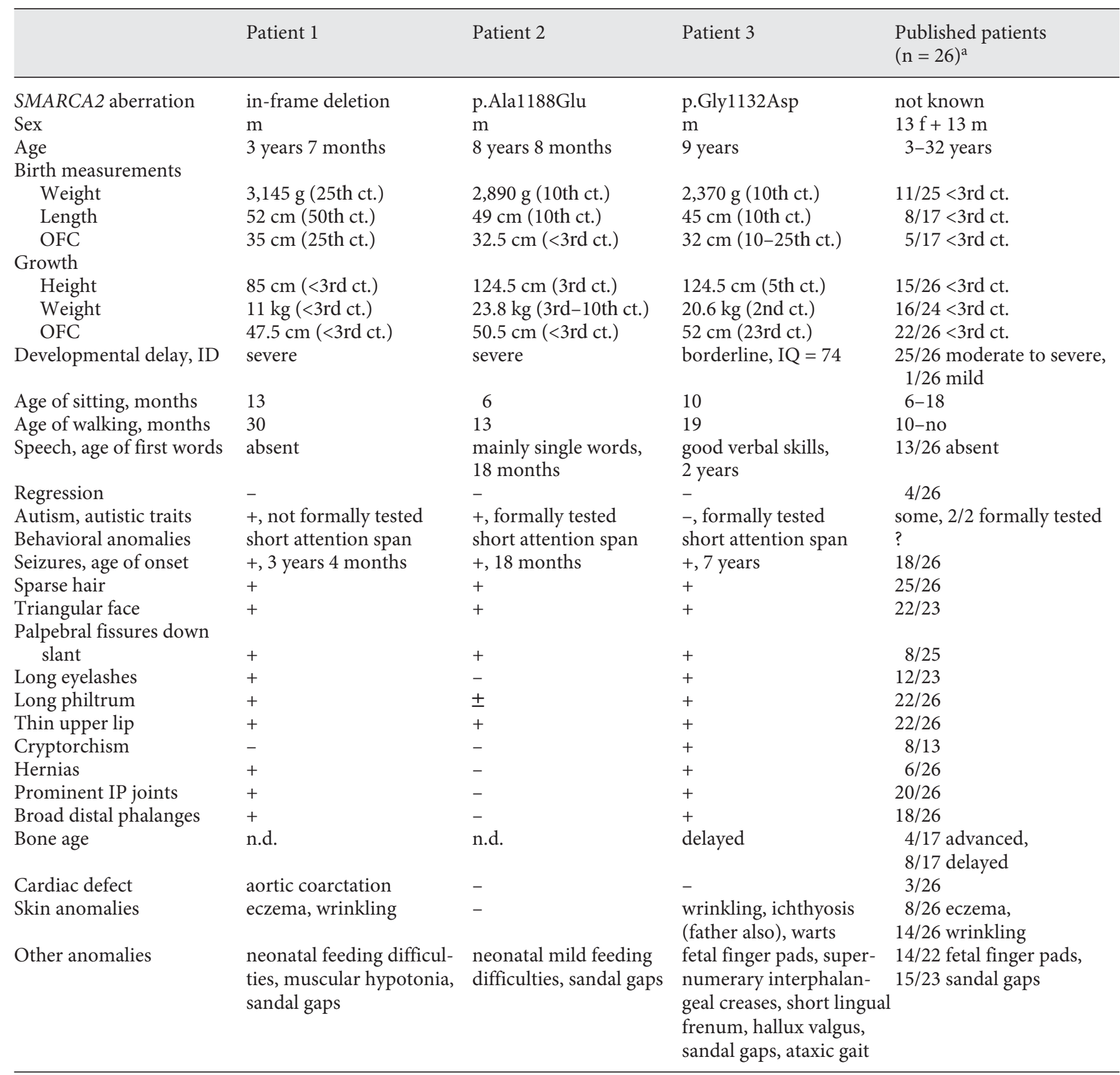

ct. = Centiles; ID = intellectual disability; IP = interphalangeal; n.d. = not determined; OFC = occipito-frontal head circumference.

a Twenty-three cases described by Sousa et al. [2009] and summarizing all published patients [Nicolaides and Baraitser, 1993; Krajewska-Walasek et al., 1996; Morin et al., 2003; Witters and Fryns, 2003; Sousa et al., 2009] plus 3 further published cases [Castori et al., 2008; Gana et al., 2011]. 
it is interesting that sparse hair as a hallmark of Nicolaides-Baraitser syndrome is not mentioned in any of the DECIPHER patients with 9 large deletions and 4 large duplications including the SMARCA2 gene for which clinical descriptions are provided. This further supports a specific role of the C-terminal helicase domain for the distinct phenotype of Nicolaides-Baraitser syndrome.

Twenty-five of 26 published patients with NicolaidesBaraitser syndrome [Nicolaides and Baraitser, 1993; Krajewska-Walasek et al., 1996; Morin et al., 2003; Witters and Fryns, 2003; Castori et al., 2008; Sousa et al., 2009; Gana et al., 2011] as well as our patients 1 and 2 showed moderate to severe mental retardation; therefore, the finding of borderline IQ in patient 3 seems rather exceptional. Of note, behavioral anomalies with short attention span and/or autistic features were observed in all of our patients and specifically reported by Gana et al. [2011] in 2 patients, thus possibly representing a so far underrecognized aspect of Nicolaides-Baraitser syndrome. Though a role of SMARCA2 and the SWI/SNF complex in cancer is supposed [Reisman et al., 2009], malignancies have not been reported in any of the patients with Nicolaides-Baraitser syndrome so far.

Individual patients with defects in either SMARCA2 or $A R I D 1 B$ show clinical overlap regarding aspects like (mild) short stature and certain facial dysmorphism such as downslanting palpebral fissures or thin upper lip. In- tellectual disability is variable in both conditions but ranging predominantly from moderate to severe. The role of both genes in the same chromatin remodeling complex might explain these clinical similarities. However, the phenotype in patients with SMARCA2 defects seems to be far more distinct, whereas ARID1B haploinsufficiency results in a more variable and less recognizable phenotype [Halgren et al., 2011; Hoyer et al., in press]. In addition, our SMARCA2 mutation-positive patients and the majority of published Nicolaides-Baraitser syndrome patients [Sousa et al., 2009; Gana et al., 2011] had epilepsy, while in patients with $A R I D 1 B$ haploinsufficiency seizures occur only rarely.

In conclusion, molecular karyotyping enabled us to identify de novo defects of the C-terminal helicase domain of SMARCA2 in patients with Nicolaides-Baraitser syndrome. In addition, we extend the phenotypic spectrum to include borderline intelligence.

\section{Acknowledgements}

We thank the patients and their families for their cooperation.

This study was supported by the German Intellectual Disability Network (MRNET) through a grant from the German Ministry of Education and Research to ARa and ARe (01GS08160), by a DFG grant to CZ (ZW 184/1-1), and by a SNF grant 320030 135669 to ARa.

\section{References}

- Amiel J, Rio M, de Pontual L, Redon R, Malan V, et al: Mutations in TCF4, encoding a class I basic helix-loop-helix transcription factor, are responsible for Pitt-Hopkins syndrome, a severe epileptic encephalopathy associated with autonomic dysfunction. Am J Hum Genet 80: 988-993 (2007).

- Castori M, Covaciu C, Rinaldi R, Grammatico P, Paradisi M: A rare cause of syndromic hypotrichosis: Nicolaides-Baraitser syndrome. J Am Acad Dermatol 59:S92-98 (2008).

de Vries BB, Pfundt R, Leisink M, Koolen DA, Vissers LE, et al: Diagnostic genome profiling in mental retardation. Am J Hum Genet 77:606-616 (2005).

-Flowers S, Nagl NG Jr, Beck GR Jr, Moran E: Antagonistic roles for BRM and BRG1 SWI/ SNF complexes in differentiation. J Biol Chem 284:10067-10075 (2009).

- Gana S, Panizzon M, Fongaro D, Selicorni A, Memo L, et al: Nicolaides-Baraitser syndrome: two new cases with autism spectrum disorder. Clin Dysmorphol 20:38-41 (2011).
Halgren C, Kjaergaard S, Bak M, Hansen C, El- Koga M, Ishiguro H, Yazaki S, Horiuchi Y, Arai Schich Z, et al: Corpus callosum abnormalities, intellectual disability, speech impairment, and autism in patients with haploinsufficiency of ARID1B. Clin Genet [Epub ahead of print] (2011).

Hoyer J, Dreweke A, Becker C, Gohring I, Thiel $\mathrm{CT}$, et al: Molecular karyotyping in patients with mental retardation using $100 \mathrm{~K}$ singlenucleotide polymorphism arrays. J Med Genet 44:629-636 (2007).

Hoyer J, Ekici AB, Endele S, Popp B, Zweier C, et al: Haploinsufficiency of $A R I D 1 B$, a member of the SWI/SNF-A chromatin-remodeling complex, is a frequent cause of intellectual disability. Am J Hum Genet (in press).

Kadam S, Emerson BM: Transcriptional specificity of human SWI/SNF BRG1 and BRM chromatin remodeling complexes. Mol Cell 11:377-389 (2003).

Kingston RE, Narlikar GJ: ATP-dependent remodeling and acetylation as regulators of chromatin fluidity. Genes Dev 13:2339-2352 (1999).
$\mathrm{M}$, et al: Involvement of SMARCA2/BRM in the SWI/SNF chromatin-remodeling complex in schizophrenia. Human Mol Genet 18:2483-2494 (2009).

Krajewska-Walasek M, Chrzanowska K, Czermiska-Kowalska A: Another patient with an unusual syndrome of mental retardation and sparse hair? Clin Dysmorphol 5:183-186 (1996). ME, Szuhai K, et al: Peters Plus syndrome is caused by mutations in B3GALTL, a putative glycosyltransferase. Am J Hum Genet 79: 562-566 (2006).

-Morin G, Villemain L, Baumann C, Mathieu M, Blanc N, et al: Nicolaides-Baraitser syndrome: confirmatory report of a syndrome with sparse hair, mental retardation, and short stature and metacarpals. Clini Dysmorphol 12:237-240 (2003).

Nicolaides P, Baraitser M: An unusual syndrome with mental retardation and sparse hair. Clin Dysmorphol 2:232-236 (1993).
Lesnik Oberstein SA, Kriek M, White SJ, Kalf 
Peterson CL, Workman JL: Promoter targeting and chromatin remodeling by the SWI/SNF complex. Curr Opin Genet Dev 10:187-192 (2000).

Reisman D, Glaros S, Thompson EA: The SWI/ SNF complex and cancer. Oncogene 28: 1653-1668 (2009).

-Saitsu H, Kato M, Mizuguchi T, Hamada K, Osa$\mathrm{ka} \mathrm{H}$, et al: De novo mutations in the gene encoding STXBP1 (MUNC18-1) cause early infantile epileptic encephalopathy. Nat Genet 40:782-788 (2008).

-Slavotinek AM: Novel microdeletion syndromes detected by chromosome microarrays. Hum Genet 124:1-17 (2008).

- Sousa SB, Abdul-Rahman OA, Bottani A, Cormier-Daire V, Fryer A, et al: NicolaidesBaraitser syndrome: delineation of the phenotype. Am J Med Genet Part A 149A:16281640 (2009).
Van Houdt JK, Nowakowska B, de Sousa SB, van Schaik BD, Seuntjes E, et al: Whole exome sequencing reveals de novo variants that affect chromatin remodelling processes in Nicolaides-Baraitser syndrome patients. Abstract \#308, presented at the 12th International Congress of Human Genetics/61st Annual Meeting of The American Society of Human Genetics, October 15th, 2011, Montreal, Canada.

-Vissers LE, van Ravenswaaij CM, Admiraal R, Hurst JA, de Vries BB, et al: Mutations in a new member of the chromodomain gene family cause CHARGE syndrome. Nat Genet 36:955-957 (2004).
Witters I, Fryns JP: Mental retardation, sparse hair, facial dysmorphism with a prominent lower lip, and lipodystrophy. A variant example of Nicolaides-Baraitser syndrome? Genet Couns 14:245-247 (2003).

Yoo AS, Crabtree GR: ATP-dependent chromatin remodeling in neural development. Curr Opin Neurobiol 19:120-126 (2009).

Zweier C, Peippo MM, Hoyer J, Sousa S, Bottani A, et al: Haploinsufficiency of TCF4 causes syndromal mental retardation with intermittent hyperventilation (Pitt-Hopkins syndrome). Am J Hum Genet 80:994-1001 (2007).

Zweier M, Gregor A, Zweier C, Engels H, Sticht $\mathrm{H}$, et al: Mutations in $M E F 2 C$ from the 5 q14.3q15 microdeletion syndrome region are a frequent cause of severe mental retardation and diminish MECP2 and CDKL5 expression. Hum Mutat 31:722-733 (2010). 